DAMTP-2005-94

hep-th/0510115

\title{
Dilaton Domain Walls and Dynamical Systems
}

\author{
Julian Sonner and Paul K. Townsend \\ Department of Applied Mathematics and Theoretical Physics, \\ University of Cambridge, \\ Centre for Mathematical Sciences, \\ Wilberforce Road, \\ Cambridge CB3 0WA, UK
}

\begin{abstract}
Domain wall solutions of $d$-dimensional gravity coupled to a dilaton field $\sigma$ with an exponential potential $\Lambda e^{-\lambda \sigma}$ are shown to be governed by an autonomous dynamical system, with a transcritical bifurcation as a function of the parameter $\lambda$ when $\Lambda<0$. All phase-plane trajectories are found exactly for $\lambda=0$, including separatrices corresponding to walls that interpolate between $a d S_{d}$ and $a d S_{d-1} \times \mathbb{R}$, and the exact solution is found for $d=3$. Janus-type solutions are interpreted as marginal bound states of these "separatrix walls". All flat domain wall solutions, which are given exactly for any $\lambda$, are shown to be supersymmetric for some superpotential $W$, determined by the solution.
\end{abstract}




\section{Introduction}

There are many supergravity models of interest for which the action can be consistently truncated to a $d$-dimensional action for metric $g_{\mu \nu}$ and dilaton field $\sigma$ with Lagrangian density

$$
\mathcal{L}=\sqrt{-\operatorname{det} g}\left[R-\frac{1}{2}(\partial \sigma)^{2}-\Lambda e^{-\lambda \sigma}\right]
$$

where $\lambda$ is a constant, which we may assume to be non-negative, and $\Lambda$ is a nonzero constant, of either sign ${ }^{1}$, that equals the cosmological constant when $\lambda=0$. Cosmological solutions for this class of models have been much studied, and are wellunderstood; in particular, a qualitative understanding of the entire space of solutions for a given $\lambda$ is made possible by the observation that the equations governing homogeneous and isotropic cosmologies define a 2-dimensional autonomous dynamical system [1].

Domain wall solutions of the same class of models have also attracted considerable attention, in part because there exist domain wall solutions that, in a supergravity context, preserve some fraction of supersymmetry ${ }^{2}$. The equations to be solved for domain walls are formally rather similar to those for cosmologies; instead of an evolution in time one has an 'evolution' in a space coordinate, and one 'evolves' a $(d-1)$-dimensional spacetime instead of a spatial hypersurface. In this paper we exploit this similarity to show that the equations for domain wall solutions can also be expressed as those of a 2-dimensional autonomous dynamical system. This again allows a qualitative understanding of the entire space of domain wall solutions, for given $\lambda$, as a set of phase-plane trajectories.

The trajectories corresponding to flat domain walls (with a Minkowski worldvolume geometry) divide the phase plane into three regions. For $\Lambda<0$, two regions for which the wall's worldvolume geometry is de Sitter $(d S)$ are separated by one for which it is anti-de Sitter $(a d S)$, and vice versa for $\Lambda>0$. All flat domain wall solutions can be found exactly [3, 4]; here we recover these results following the method used in [5] to find all flat cosmological solutions (first given in [6]). The qualitative behaviour of all other trajectories, corresponding to walls with $d S$ or adS worldvolume geometry, is determined by the positions and the nature of the fixed points. The analysis is essentially the same as the cosmological case but the spacetime interpretation of the solutions is of course different.

The $\lambda=0$ case is special, and of particular interest in that the full spacetime, and not just the domain wall's worldvolume, can be de Sitter or anti-de Sitter. For this case, we find all phase-plane trajectories exactly. These include two fixed points that each correspond to the $a d S_{d}$ vacuum, foliated by Minkowski spaces. Other trajectories correspond to the same $a d S_{d}$ vacuum but foliated by $d S$ or $a d S$ spaces.

\footnotetext{
${ }^{1}$ A shift of $\sigma$ has the effect of scaling $\Lambda$ when $\lambda \neq 0$, so only the sign of $\Lambda$ is physically relevant, but the magnitude of $\Lambda$ is physically relevant when $\lambda=0$.

${ }^{2}$ The issue of supersymmetry preservation for dilaton domain walls appears to have been first adressed in 2, albeit in a more general model with domain wall solutions that differ from those discussed here.
} 
The trajectory corresponding to the $a d S_{d-1}$ foliation of $a d S_{d}$ is actually a special case of a one-parameter family of trajectories that interpolate between the two $a d S_{d}$ fixed points. These correspond to the "Janus" solutions of [7. A limit of these Janus trajectories yields the union of two separatrix trajectories, each interpolating between one of the $a d S_{d}$ fixed points and one of two other fixed points, each of which corresponds to an $a d S_{d-1} \times \mathbb{R}$ solution (the "curious linear dilaton" solution of [7]). These separatrices correspond to new solutions, which we call "separatrix walls", and we find the exact separatrix wall solution for $d=3$. We point out that Janus solutions can be interpreted as marginal bound states of separatrix walls.

For $\lambda>0$ our results are more qualitative, although all fixed-point (in addition to flat) domain wall solutions can be found exactly, and all trajectories can be found exactly in the $\lambda \rightarrow \infty$ limit. One interesting feature of the family of $\Lambda<0$ phase plane trajectories, parametrized by $\lambda$, is that a bifurcation occurs at a critical value $\lambda_{c}$ of $\lambda$. Here we show that this is, in the language of dynamical systems, a transcritical bifurcation. Another interesting feature is that for $\lambda<\lambda_{c}$ there is a one-parameter family of Janus-type solutions that are similarly "two-faced" but which are asymptotic to a $\lambda$-deformation of $a d S_{d}$.

As already mentioned, one reason for interest in domain wall solutions of our model is that, in the supergravity context, domain walls may preserve some fraction of the supersymmetry of the supergravity vacuum. This issue has been investigated previously in the context of various specific supergravity models that have a consistent truncation to (1.1); we will comment later on how this work fits in with our results, which are model independent in the following sense. On general grounds [8, 9, 10] one expects the potential $V(\sigma)$ for any single scalar field to take the form

$$
V=2\left[\left(W^{\prime}\right)^{2}-\alpha^{2} W^{2}\right] \quad\left(W^{\prime} \equiv d W / d \sigma\right)
$$

for some super-potential function $W(\sigma)$. Locally, one can view this as a differential equation that determines $W$ for given $V$ [11, but the global situation is more subtle. In our case, for which $V=\Lambda e^{-\lambda \sigma}$, we will see that (1.2) does not always determine a unique superpotential, so that it is possible for a domain wall solution to be supersymmetric for one choice of superpotential and not for another. We shall say that a domain wall solution is "supersymmetric" if it preserves supersymmetry for some choice of superpotential. Remarkably, we find that the possible superpotentials correspond to the possible flat domain wall solutions, and that any flat domain wall is supersymmetric for a choice of superpotential that is actually determined by the solution!

Essentially the same point is made in [7], as we learnt after submission to the archives of an earlier version of this paper. The conclusions of [7] on the supersymmetry of flat walls apply for a general scalar potential $V$, but those presented here for exponential potentials are more complete. For curved walls, we find that the only "supersymmetric" solutions are the $d S_{d-1}$ and $a d S_{d-1}$ foliations of $a d S_{d}$, but a larger class of curved "supersymmetric" domain walls is found in [7] by allowing for a matrix-valued superpotential. We comment further on this later but otherwise leave to the reader any more detailed comparison with [7]. 
We begin by introducing the constants

$$
\alpha=\sqrt{\frac{(d-1)}{2(d-2)}}, \quad \beta=\frac{1}{\sqrt{2(d-1)(d-2)}} .
$$

Now consider the domain wall ansatz

$$
d s_{d}^{2}=e^{2 \alpha \varphi} f^{2}(z) d z^{2}+e^{2 \beta \varphi} d \Sigma_{k}^{2}, \quad \sigma=\sigma(z)
$$

for arbitrary function $f(z)$, where $d \Sigma_{k}^{2}$ is the metric of a $(d-1)$-dimensional homogeneous spacetime with inverse radius of curvature equal to $k$; the scalar curvature is therefore $k(d-1)(d-2)$. As in the cosmological case, we may restrict to $k=-1,0,1$ without loss of generality. The isometry group is $S O(d, 1)$ for $k=1, I S O(d-1)$ for $k=0$, and $S O(d-2,2)$ for $k=-1$. Thus, $d \Sigma_{k}^{2}$ is the metric of a "unit-radius" de Sitter space for $k=1$, a Minkowski space for $k=0$ and a "unit-radius" anti-de Sitter space for $k<0$; note that the domain wall is flat for $k=0$ but curved for $k \neq 0$. This ansatz yields the effective Lagrangian ${ }^{3}$

$$
L=\frac{1}{2 f}\left[\dot{\varphi}^{2}-\dot{\sigma}^{2}\right]+f\left[k(d-1)(d-2) e^{\varphi / \alpha}-\Lambda e^{2 \alpha \varphi-\lambda \sigma}\right],
$$

where the overdot indicates a derivative with respect to $z$. This can be interpreted as a reparametrization-invariant Lagrangian for a relativistic particle with a 'time'dependent potential energy in a 2-dimensional Minkowski spacetime.

\section{The dynamical system}

If we fix the $z$-reparametrization invariance by choosing

$$
f(z)=e^{\lambda \sigma / 2-\alpha \varphi}
$$

then the equations of motion for $(\varphi, \sigma)$ and $f$ become equivalent to the equations

$$
\begin{aligned}
\ddot{\sigma} & =\frac{1}{2} \lambda \dot{\sigma}^{2}-\alpha \dot{\varphi} \dot{\sigma}-\lambda \Lambda \\
\ddot{\varphi} & =\frac{1}{2} \lambda \dot{\sigma} \dot{\varphi}-\beta \dot{\varphi}^{2}-\frac{1}{2 \alpha} \dot{\sigma}^{2}-2 \beta \Lambda,
\end{aligned}
$$

together with the constraint

$$
\dot{\varphi}^{2}-\dot{\sigma}^{2}+2 \Lambda=\frac{k}{\beta^{2}} e^{\lambda \sigma-2 \beta \varphi} .
$$

\footnotetext{
${ }^{3}$ Apart from a minor change of notation, and the interpretation of the independent variable, this is identical to the Lagrangian obtained in [12] for the cosmological case; its domain-wall interpretation appeared in unpublished notes of E. Bergshoeff, A. Collinucci and D. Roest that were preliminary to that work. For either interpretation, it can be verified directly that solutions of the effective Lagrangian yield solutions of the equations of motion of (1.1).
} 
We note here that the domain wall metric for the choice (2.1) is

$$
d s_{d}^{2}=e^{\lambda \sigma(z)} d z^{2}+e^{2 \beta \varphi(z)} d \Sigma_{k}^{2}
$$

Equations (2.2) define a 2-dimensional autonomous dynamical system, with coordinates $(\dot{\sigma}, \dot{\varphi})$. The entire space of phase-plane trajectories is determined by the positions and nature of the fixed points. For $k=0$ the constraint (2.3) becomes the hyperbola

$$
\dot{\varphi}^{2}-\dot{\sigma}^{2}=-2 \Lambda
$$

and the two branches of this hyperbola divide the phase plane into three regions. For $\Lambda<0$ there is a 'central' $k=-1$ region containing the line $\dot{\varphi}=0$ that separates two $k=1$ regions. For $\Lambda>0$ there is a central $k=1$ region containing the line $\dot{\sigma}=0$ that separates two $k=-1$ regions. For $k \neq 0$ the constraint merely determines the value of $\lambda \sigma-2 \beta \varphi$ at a given point on a phase-plane trajectory, so it has no effect on the trajectories themselves. We shall therefore concentrate on the equations (2.2). In the notation

$$
u=\dot{\sigma} \quad v=\dot{\varphi}
$$

these two equations become

$$
\begin{aligned}
\dot{u} & =\frac{1}{2} \lambda u^{2}-\alpha u v-\lambda \Lambda \\
\dot{v} & =\frac{1}{2} \lambda u v-\beta v^{2}-\frac{1}{2 \alpha} u^{2}-2 \beta \Lambda .
\end{aligned}
$$

The autonomous dynamical system defined by these equations differs from the one that governs FLRW cosmologies only by a flip of the signs of $\Lambda$ and $k$. Thus, the domain wall trajectories for negative $\Lambda$ are the same as the cosmological trajectories for positive $\Lambda$, and vice-versa, but with the opposite sign of $k$ in each case.

\subsection{Fixed points}

As with any autonomous dynamical system, the first task is to identify the fixed points, in this case the points in the $(u, v)$ plane at which $(\dot{u}, \dot{v})=(0,0)$. Following the analysis of [13] for the cosmological case, we proceed from the observation that the fixed points are such that

$$
(\lambda u-2 \beta v)(\lambda v-2 \alpha u)=0 .
$$

There are thus two types of fixed point:

- Type 1: $u=\frac{\lambda}{2 \alpha} v$. In this case the fixed point conditions can be satisfied only if $\lambda \neq 2 \alpha$. Given this, one finds that

$$
v^{2}=\frac{8 \alpha^{2}}{\lambda^{2}-4 \alpha^{2}} \Lambda
$$


which implies that a fixed point exists for $\Lambda<0$ iff $\lambda<2 \alpha$ and for $\Lambda>0$ iff $\lambda>2 \alpha$. Thus, at this type of fixed point,

$$
u= \pm \lambda K(\lambda), \quad v= \pm 2 \alpha K(\lambda)
$$

where

$$
K(\lambda)=\sqrt{\left|\frac{2 \Lambda}{\lambda^{2}-4 \alpha^{2}}\right|} .
$$

It follows that

$$
v^{2}-u^{2}=2|\Lambda|
$$

at the fixed point, and hence that that these fixed points lie on the $k=0$ hyperbola; in fact, these fixed points come in pairs, one on each branch of the $k=0$ hyperbola.

- Type 2: $v=\frac{\lambda}{2 \beta} u$. In this case the fixed point conditions are satisfied when

$$
u^{2}=-\frac{2}{d-2} \Lambda
$$

which shows that $\Lambda$ must be negative. Thus, at this type of fixed point,

$$
u= \pm \lambda_{c} \sqrt{|\Lambda|}, \quad v= \pm \lambda \sqrt{(d-1)|\Lambda|}
$$

where

$$
\lambda_{c}=\sqrt{\frac{2}{d-2}} .
$$

Again, each fixed point of this type occurs in pairs, one on each of the lines $|u|=\lambda_{c} \sqrt{|\Lambda|}$. The constraint (2.3) now yields

$$
\left(\lambda^{2}-\lambda_{c}^{2}\right)|\Lambda|=2(d-2) k e^{\lambda \sigma-2 \beta \varphi},
$$

which shows that $k=-1$ for $\lambda<\lambda_{c}$ and $k=1$ for $\lambda>\lambda_{c}$. For $\lambda=\lambda_{c}$ this fixed point coincides with the one on the $k=0$ hyperbola.

To summarize, fixed points occur for $\Lambda>0$ only if $\lambda>2 \alpha$, and then only for $k=0$, with one fixed point on each branch of the $k=0$ hyperbola. A similar pair of $k=0$ fixed points occurs for $\Lambda<0$ when $\lambda<2 \alpha$, but there is also another pair of fixed points, with $k=-1$ for $\lambda<\lambda_{c}$ and $k=1$ for $\lambda>\lambda_{c}$. For $\lambda=\lambda_{c}$, each of these fixed points coincides with one of the pair of $k=0$ fixed points. This implies a bifurcation in the family of dynamical systems parametrized by $\lambda$. We now turn to an investigation of the nature of this bifurcation. 


\subsection{The transcritical bifurcation}

We now concentrate on the case of $\Lambda<0$, and for simplicity we set $\Lambda=-1$. As we are interested in what happens when $\lambda \approx \lambda_{c}$, we define

$$
\mu=\lambda-\lambda_{c}
$$

as a new parameter. Also, for later convenience, we define

$$
J(\mu) \equiv \sqrt{(d-2)(d-10) \lambda^{2}+16}=(d-2) \lambda_{c}+\mathcal{O}(\mu)
$$

To investigate the nature of the bifurcation at $\mu=0$, it is convenient to introduce the shifted variables

$$
u=\lambda_{c}+\tilde{u}, \quad v=\sqrt{d-1} \lambda+\tilde{v} .
$$

The $k=-1$ fixed point in the $u>0$ half-plane is now at $(\tilde{u}, \tilde{v})=(0,0)$ for any value of $\mu$. To put the equations into a standard form in a neigbourhood of this fixed point, we introduce the new variables

$$
x=\tilde{v}+s_{-} \tilde{u}, \quad y=\tilde{v}+s_{+} \tilde{u},
$$

where

$$
s_{ \pm}=\frac{1}{4 \alpha}[(d-4) \lambda \pm J]
$$

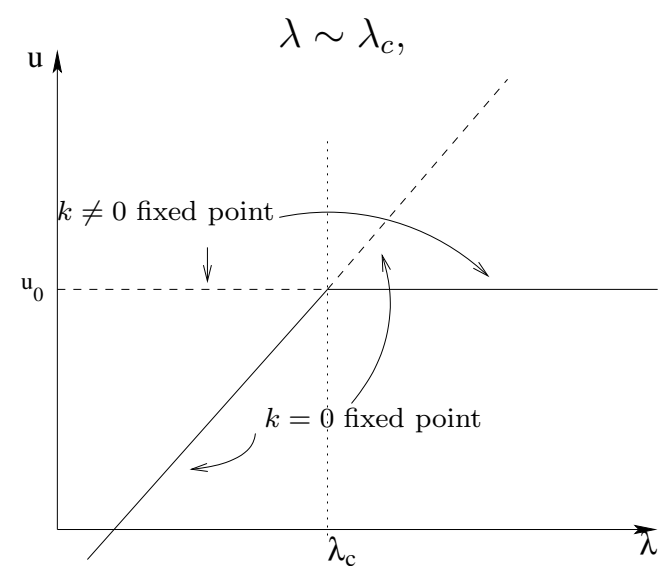

Figure 1: Bifurcation Diagram. The transcritical bifurcation corresponds to an exchange of stable (solid line) and unstable (dashed line) directions between two fixed points.

One then finds that the equations (2.7) take the form ${ }^{4}$

$$
\begin{aligned}
& \dot{x}=A(\mu) x+F_{1}(x, y, \mu), \\
& \dot{y}=-B(\mu) y+F_{2}(x, y, \mu),
\end{aligned}
$$

\footnotetext{
${ }^{4}$ This is a standard form, as given in (for example) Chapter 8 of [14, which we found to be a useful reference.
} 
where $B$ is positive, $A(0)$ vanishes, and $\left(F_{1}, F_{2}\right)$ are two functions that both vanish and have vanishing first-derivatives with respect to $x, y$ and $\mu$ at $(x, y, \mu)=(0,0,0)$. Specifically, one finds that

$$
A=\frac{1}{4} \lambda_{c}\left[J-(d-2) \lambda_{c}\right], \quad B=\frac{1}{4} \lambda_{c}\left[J+(d-2) \lambda_{c}\right]
$$

and that

$$
\begin{aligned}
F_{1}= & \frac{\beta}{2 J^{2}}\left\{-\left[4(3 d-1)-(d+8)(d-2) \lambda^{2}+3(d-2) \lambda J\right] x^{2}\right. \\
& +\left[8(d-3)-(d-1)(d-6)(d-2) \lambda^{2}+(d-1)(d-2) \lambda J\right] x y \\
& \left.+\left[4(d-3)+(d-2)\left(d^{2}-10 d+18\right) \lambda^{2}-(d-4)(d-2) \lambda J\right] y^{2}\right\} \\
F_{2}= & \frac{\beta}{2 J^{2}}\left\{\left[4(d-3)+(d-2)\left(d^{2}-10 d+18\right) \lambda^{2}+(d-4)(d-2) \lambda J\right] x^{2}\right. \\
& +\left[8(d-3)-(d-1)(d-6)(d-2) \lambda^{2}-(d-1)(d-2) \lambda J\right] x y \\
& \left.-\left[4(3 d-1)-(d+8)(d-2) \lambda^{2}-3(d-2) \lambda J\right] y^{2}\right\}
\end{aligned}
$$

Expansion in powers of $\mu$ yields

$$
A(\mu)=-2 \mu \lambda_{c}+\mathcal{O}\left(\mu^{2}\right), \quad B(\mu)=1+\frac{1}{2}(d-6) \mu \lambda_{c}+\mathcal{O}\left(\mu^{2}\right),
$$

which confirms that the fixed point at the origin is hyperbolic for $\mu \neq 0$ but nonhyperbolic for $\mu=0$, and

$$
\begin{aligned}
& F_{1}=\beta\left[-4 x^{2}+4 x y-y^{2}\right]+\mathcal{O}(\mu) \\
& F_{2}=\beta\left[(d-5) x^{2}-(d-5) x y-y^{2}\right]+\mathcal{O}(\mu),
\end{aligned}
$$

where the $\mathcal{O}(\mu)$ terms in $\left(F_{1}, F_{2}\right)$ are quadratic in $(x, y)$, so that the functions $\left(F_{1}, F_{2}\right)$ are $\mu$-independent to quadratic order.

The behaviour near $\mu=0$ is determined by the dynamics on a 2-dimensional 'extended centre manifold', this being the centre manifold of the extended system in which $\mu$ is taken as a third variable with the trivial equation $\dot{\mu}=0$. The extended centre manifold is given by $y=h(x, \mu)$ for some function $h$ that both vanishes and has vanishing first-derivatives with respect to $x$ and $\mu$ at $(x, \mu)=(0,0)$. The function $h$ can be found as a power series in $x$ and $\mu$ by requiring consistency with the evolution equations (2.22). This yields

$$
h(x, \mu)=(d-5) \alpha x^{2}+\ldots,
$$

where the dots indicate terms at least cubic in the two variables $(x, \mu)$; note that $h$ is independent of $\mu$ at quadratic order, which is all that we will need. Substitution of $y=h(x, \mu)$ into the equation for $x$ yields an equation of the form

$$
\dot{x}=G(x, \mu), \quad G=-2 \mu \lambda_{c} x-4 \beta x^{2}+\ldots .
$$


In terms of the rescaled variable $w$ and the rescaled parameter $\nu$, defined by

$$
w=4 \beta x, \quad \nu=-2 \mu \lambda_{c}
$$

this equation takes the form

$$
\dot{w}=\nu w-w^{2}+\ldots
$$

This is the standard form for a transcritical bifurcation in which the stability properties of the fixed points are exchanged as they cross at $\nu=0$, as illustrated in the bifurcation diagram of Fig. 1. Indeed, the $k=0$ fixed point is stable for $\lambda<\lambda_{c}$ and unstable for $\lambda>\lambda_{c}$, while the reverse is true for the $k=-1$ fixed point.

\section{Domain walls for $\lambda=0$}

For $\lambda=0$ the equations (2.7) become

$$
\dot{u}=-\alpha u v, \quad \dot{v}=-\beta v^{2}-\frac{1}{2 \alpha} u^{2}-2 \beta \Lambda,
$$

and the constraint (2.3) becomes

$$
\beta^{2}\left(v^{2}-u^{2}+2 \Lambda\right)=k e^{-2 \beta \varphi} .
$$

We shall first consider the special solutions obtained by setting $u \equiv 0$. We then obtain the exact phase-plane trajectories for all solutions, and present an exact solution corresponding to a separatrix trajectory.

\subsection{Some special solutions}

For $u \equiv 0$, the equations (3.1) reduce to

$$
\dot{v}=-\beta\left(v^{2}+2 \Lambda\right)
$$

For $\Lambda<0$ there are three solutions, with $k=0,-1,1$ :

- $k=0$. This is the fixed point solution with $v^{2}=2|\Lambda|$, and hence $\varphi=\sqrt{2|\Lambda|} z+$ $\varphi_{0}$ for constant $\varphi_{0}$, which we may set to zero without loss of generality, so the domain wall metric is

$$
d s_{d}^{2}=d z^{2}+e^{2 \beta \sqrt{2|\Lambda|} z} d s_{d-1}^{2}(\text { Mink }) .
$$

where "Mink" indicates a Minkowski metric. This is just $a d S_{d}$ foliated by Minkowski hypersurfaces. For standard Minkowski coordinates, this yields $a d S_{d}$ in horospherical coordinates. 
- $k=-1$. In this case $|v|<\sqrt{2|\Lambda|}$ and

$$
v=\sqrt{2|\Lambda|} \tanh (\beta \sqrt{2|\Lambda|} z) .
$$

The constraint (3.2) becomes

$$
e^{-2 \beta \varphi}=\beta^{2}\left(2|\Lambda|-v^{2}\right)=\frac{2 \beta^{2}|\Lambda|}{\cosh ^{2}(\beta \sqrt{2|\Lambda|} z)},
$$

and hence the metric is

$$
d s_{d}^{2}=d z^{2}+\frac{1}{2 \beta^{2}|\Lambda|} \cosh ^{2}(\beta \sqrt{2|\Lambda|} z) d s_{d-1}^{2}(a d S) .
$$

This is $a d S_{d}$ foliated by anti-de Sitter hypersurfaces; the $d=5$ case is wellknown [15, 16.

- $k=1$. In this case $|v|>\sqrt{2|\Lambda|}$ and

$$
v=\sqrt{2|\Lambda|} \operatorname{coth}(\beta \sqrt{2|\Lambda|} z) .
$$

The constraint (3.2) becomes

$$
e^{-2 \beta \varphi}=\beta^{2}\left(v^{2}-2|\Lambda|\right)=\frac{2 \beta^{2}|\Lambda|}{\sinh ^{2}(\beta \sqrt{2|\Lambda| z})},
$$

and hence the metric is

$$
d s_{d}^{2}=d z^{2}+\frac{1}{2 \beta^{2}|\Lambda|} \sinh ^{2}(\beta \sqrt{2|\Lambda|} z) d s_{d-1}^{2}(d S)
$$

This is $a d S_{d}$ foliated by de Sitter hypersurfaces [11, 17].

Thus, just as de Sitter space can be viewed as an FLRW cosmology for which spatial sections can have zero, positive or negative curvature, so anti-de Sitter space can be viewed as a domain wall spacetime for which the wall has zero, positive or negative curvature. In other words, $d$-dimensional anti de Sitter space can be foliated by $(d-1)$-dimensional leaves with Minkowski $(k=0)$, de Sitter $(k=1)$ or anti-de Sitter $(k=-1)$ geometry.

Continuing with this analogy, we observe that since anti de Sitter space can be viewed as an FLRW cosmology with $k=-1$ (but not for $k=0$ or $k=1$ ) we would expect de Sitter space to appear as a domain wall for $k=1$ (but not for $k=0$ or $k=-1$ ). Indeed, for $\Lambda>0$ there is a solution of (3.3) only if $k=1$, and this solution is

$$
v=\sqrt{2 \Lambda} \cot (\beta \sqrt{2 \Lambda} z) .
$$


The constraint (3.2) is now

$$
e^{-2 \beta \varphi}=\beta^{2}\left(v^{2}+2 \Lambda\right)=\frac{2 \beta^{2} \Lambda}{\sin ^{2}(\beta \sqrt{2 \Lambda} z)},
$$

and hence the metric is

$$
d s_{d}^{2}=d z^{2}+\frac{1}{2 \beta^{2}|\Lambda|} \sin ^{2}(\beta \sqrt{2 \Lambda} z) d s_{d-1}^{2}(d S) .
$$

This is de Sitter space foliated by de Sitter hypersurfaces [18].

Finally, returning to $\Lambda<0$, we consider the $k=-1$ fixed point at $v=0$ and

$u=\lambda_{c} \sqrt{|\Lambda|}$. This has $\varphi=\varphi_{0}$ for constant $\varphi_{0}$, which is determined by the constraint (3.2) to be such that

$$
e^{-2 \beta \varphi_{0}}=\frac{|\Lambda|}{(d-2)^{2}}
$$

The metric is therefore

$$
d s_{d}^{2}=d z^{2}+\frac{(d-2)^{2}}{|\Lambda|} d s_{d-1}^{2}(a d S) .
$$

This is a cylindrical spacetime with a $(d-1)$-dimensional anti-de Sitter cross-section: It is the domain wall analog of the Einstein Static Universe. It is also the "curious linear dilaton" solution found in [7].

\subsection{Exact phase-plane trajectories}

For either sign of $\Lambda$ the phase space trajectories may be found exactly by the method used in [13] to find the cosmological trajectories for $\Lambda<0$. From (3.1) it follows that

$$
\left(\beta v^{2}+\frac{1}{2 \alpha} u^{2}+2 \beta \Lambda\right) d u-\alpha u v d v=0
$$

on any trajectory. The left hand side is not an exact differential but if $u>0$ then the function $u^{-(d+1) /(d-1)}$ is an integrating factor and this leads to the conclusion that

$$
v^{2}-u^{2}+2 \Lambda=-c u^{2 /(d-1)}
$$

for some constant $c$. A sketch of the phase plane shows that there are no trajectories on which $u$ changes sign, and also that all trajectories with $u<0$ are mirror images of those with $u>0$, so we may restrict the discussion to follow to $u>0$. From a comparison of (3.17) with the constraint (2.3) we learn that

$$
c u^{2 /(d-1)}=-\frac{k}{\beta^{2}} e^{-2 \beta \varphi} .
$$

This determines the value of $\varphi$ at any given point on a trajectory specified by the constant $c$, except on the $k=0$ trajectories, which are obtained by the choice $c=0$, and the trajectories with $u \equiv 0$, which correspond to $|c|=\infty$. Note that

$$
\operatorname{sign} c=-k .
$$


For $\Lambda>0$ the interpretation of (3.17) is straightforward. Each trajectory with $u>0$ corresponds to one choice of $c$, with $k=1$ for $c<0$ and $k=-1$ for $c>0$. The phase-plane plot is shown in Fig. 2b.

For $\Lambda<0$ the interpretation of (3.17) is not so straightforward because of the fixed points, as shown in the phase-plane plot in Fig 2a. Observe that (3.17) is solved for any $c$ by $(u, v)=(0, \pm \sqrt{2|\Lambda|})$, which are the $k=0$ fixed points, but a sketch of the phase plane shows that there are $k=-1$ trajectories that do not have any fixed point as a limit point. The resolution of this puzzle is that a solution of (3.17) for given $c$ may have more than one branch; in other words, each value of $c$ may yield more than one trajectory. For $c<0$ this is trivially true because no such trajectory passes through $v=0$; each trajectory with $v>0$ therefore has a mirror image with $v<0$. The same is true for $c>0$ provided $c<\bar{c}$, where

$$
\bar{c}=(d-1)\left(\frac{2|\Lambda|}{d-2}\right)^{(d-2) /(d-1)} .
$$

In such cases we may restrict attention to the quadrant of the phase plane with $u<0$ and $v>0$, in which the curve (3.17) specifies a unique trajectory for given $c<\bar{c}$. In contrast, the $c>\bar{c}$ trajectories pass through $v=0$, and for these one must allow for both positive and negative $v$. For a given value of $c>\bar{c}$, the curve (3.17) has two branches in the $u>0$ half-plane. On one branch the trajectory is asymptotic to both branches of the $k=0$ hyperbola. On the other branch, the trajectory has limit points at the $k=0$ fixed points. As these fixed points correspond to $a d S_{d}$ spacetimes, the interpolating trajectories correspond to solutions that are asymptotic to $a d S_{d}$ in either of two directions. These "two-faced" solutions were called "Janus" solutions in [7] (by analogy with the Janus solution of IIB supergravity [16]).

For $c=\bar{c}$, and $u>0$, (3.17) describes the four separatrices that meet at the $v=0$ fixed point. One of these interpolates between this fixed point and the $u=0$ fixed point with $v>0$. This separatrix trajectory is therefore one of the curves described by the equation

$$
v^{2}=u^{2}+2|\Lambda|-(d-1)\left(\lambda_{c}^{2}|\Lambda|\right)^{(d-2) /(d-1)} u^{2 /(d-1)} .
$$

\subsection{An exact separatrix solution}

Let us consider in more detail the $d=3$ case, for which we may write (3.17) as

$$
v^{2}-(u-c / 2)^{2}=-\frac{1}{4}\left(c^{2}+8 \Lambda\right)
$$

For $\Lambda<0$ there is clearly a change of behaviour of the trajectories when $c^{2}=8|\Lambda|$, and for $u>0$ this occurs when $c=\sqrt{8|\Lambda|} \equiv \bar{c}$. At this critical value of $c$, the equation (3.22) degenerates to

$$
v^{2}=(u-\sqrt{2|\Lambda|})^{2}
$$




$$
\Lambda<0, \lambda=0
$$

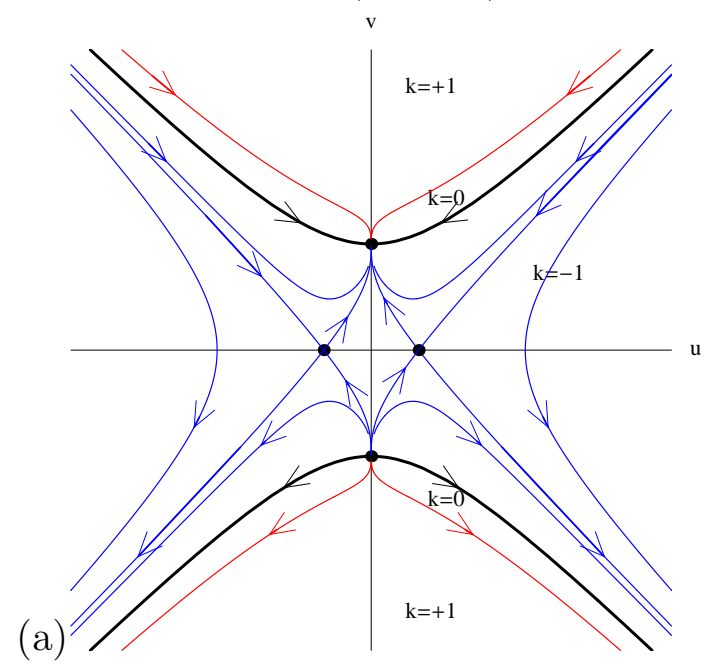

$\Lambda>0, \lambda=0$,

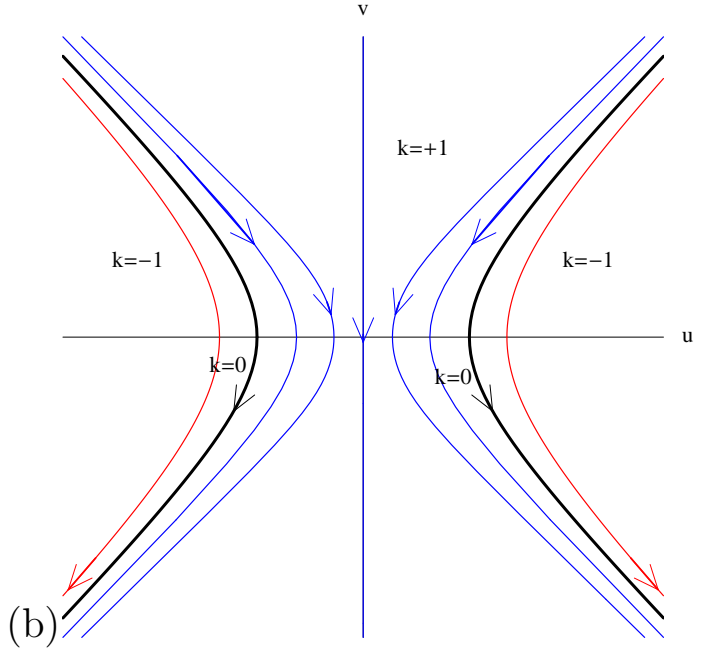

Figure 2: (a) The phase plane for $\Lambda<0$. There are four fixed points, connected by separatrices. The solutions corresponding to trajectories along the $v$-axis are foliations of $a d S$. (b) The phase plane for $\Lambda>0$. The straight line trajectory along the $v$-axis corresponds to the $d S$ foliation of de Sitter space (3.13).

This describes four straight-line separatrices that meet at the $(u, v)=(\sqrt{2|\Lambda|}, 0)$ fixed point. In particular the separatrix that interpolates between this fixed point and the $(u, v)=(0, \sqrt{2|\Lambda|})$ fixed point is the straight line

$$
u+v=\sqrt{2|\Lambda|} .
$$

On this line, the first of equations (3.1) becomes

$$
\dot{u}=-u(\sqrt{2|\Lambda|}-u) .
$$

This equation is easily integrated; taking into account that $u<\sqrt{2|\Lambda|}$ on the separatrix, we find that

$$
(u, v)=\frac{\sqrt{2|\Lambda|}}{e^{\sqrt{2|\Lambda|} z}+1}\left(1, e^{\sqrt{2|\Lambda|} z}\right) .
$$

As $k=-1$ on the separatrix, the constraint (3.2) implies that

$$
e^{\varphi}=\frac{4}{u^{2}-v^{2}+2|\Lambda|}=|\Lambda|^{-1}\left(1+e^{\sqrt{2|\Lambda|} z}\right)
$$

and hence that the metric is

$$
d s_{d}^{2}=d z^{2}+|\Lambda|^{-1}\left(1+e^{\sqrt{2|\Lambda|} z}\right) d \Sigma_{-1}^{2} .
$$


To complete the solution, we observe that (3.24) implies $\dot{\sigma}=\sqrt{2|\Lambda|}-\dot{\varphi}$, and hence that

$$
e^{\sigma-\sigma_{0}}=\left[1+e^{-\sqrt{2|\Lambda|} z}\right]^{-1}
$$

for some constant $\sigma_{0}$.

Thus, for $d=3$, we have found the exact separatrix solution, and not merely the exact phase-plane trajectory. As $z \rightarrow-\infty$ we have

$$
\varphi \sim-\log |\Lambda|, \quad \sigma \sim \sigma_{0}+\sqrt{2|\Lambda|} z,
$$

which yields the $a d S_{2} \times \mathbb{R}$ solution at the $k=-1$ fixed point. As $z \rightarrow \infty$ we have

$$
\varphi \sim \sqrt{2|\Lambda|} z-\log |\Lambda|, \quad \sigma \sim \sigma_{0},
$$

which yields the $a d S_{3}$ solution at the $k=0$ fixed point.

\section{Domain walls for $\lambda>0$}

$$
\Lambda<0, \lambda<\lambda_{c}
$$

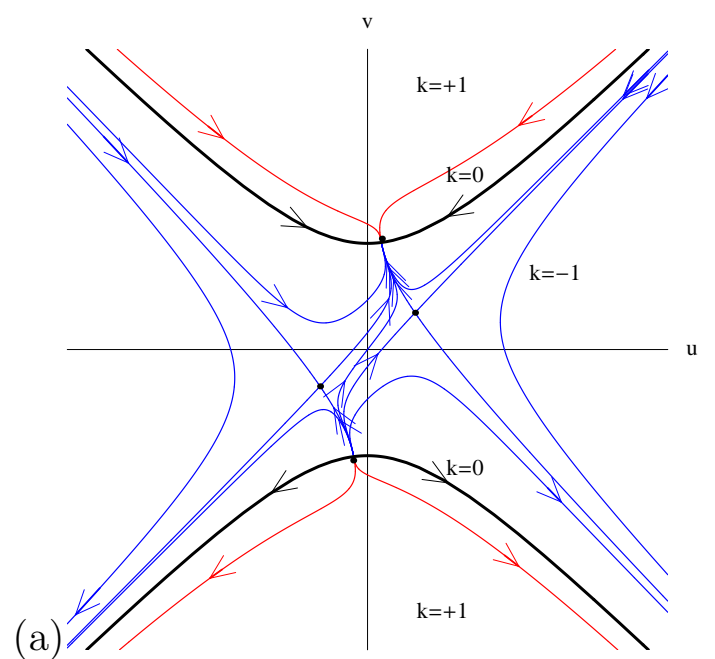

$$
\Lambda<0, \lambda=\lambda_{c}
$$

(b)

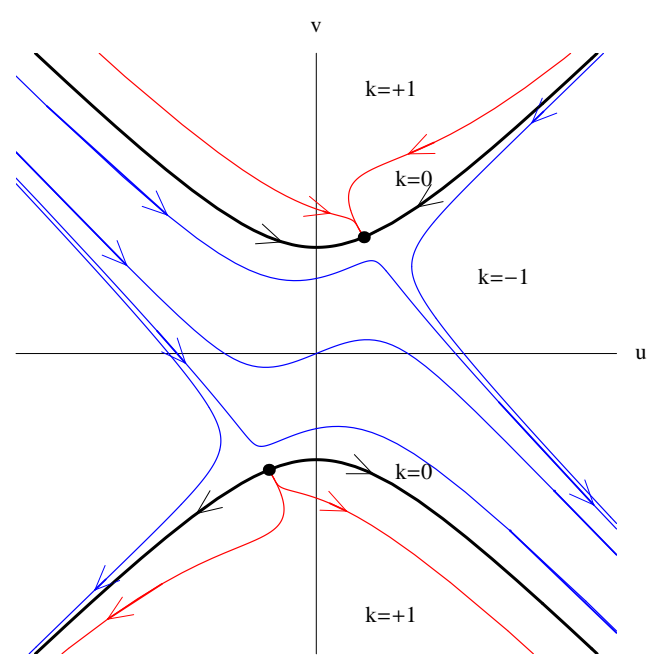

Figure 3: (a) The phase plane has the same topology as for $\lambda=0$, with four hyperbolic fixed points, but the lateral symmetry is lost. (b) The four fixed points have coalesced to form a pair of non-hyperbolic fixed points.

For $\lambda>0$ our first task is to identify the nature of the domain wall spacetimes corresponding to the fixed points. We then find exactly all $k=0$ solutions, following the method used in [5] for cosmology. For generic $k=0$ trajectories we fall back on a qualitative analysis of the phase-plane; see Figs. 3,4,5. 

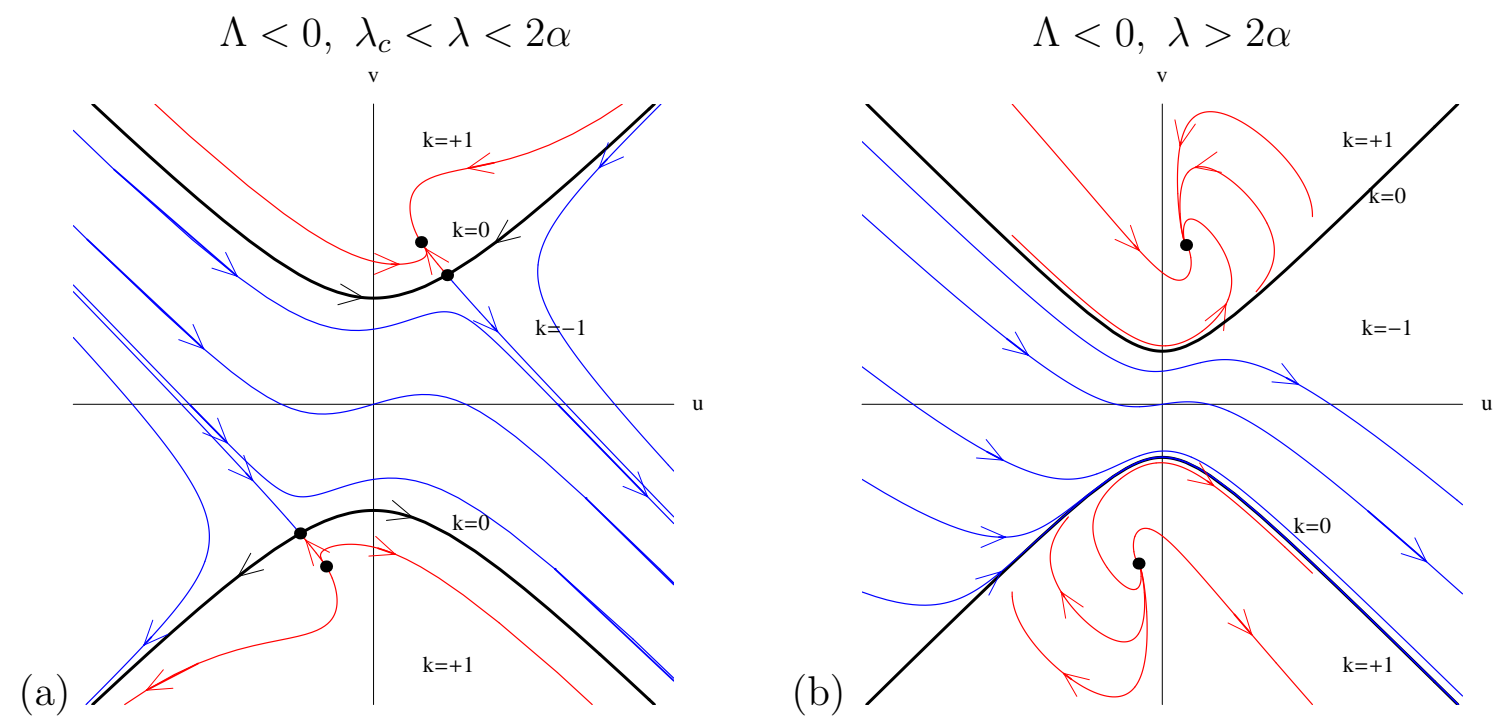

Figure 4: (a) The two $k \neq 0$ fixed points are now in the $k=-1$ regions. (b) The $k=0$ fixed points have disappeared to $\infty$.

\subsection{Fixed point solutions}

We need consider only the fixed points with $u>0$. We consider each of the types of fixed point in turn.

- Type 1. At this fixed point we have

$$
\sigma=\lambda K(\lambda) z+\sigma_{0}, \quad 2 \beta \varphi=\lambda_{c}^{2} K(\lambda) z+2 \beta \varphi_{0}
$$

for constants $\sigma_{0}$ and $\varphi_{0}$. Without loss of generality, we may choose

$$
\sigma_{0}=\frac{2}{\lambda} \log \left(\lambda^{2} K / 2\right), \quad \varphi_{0}=0
$$

in which case the fixed-point solution is

$$
d s_{d}^{2}=d r^{2}+r^{2 \lambda_{c}^{2} / \lambda^{2}} d s_{d-1}^{2}(\text { Mink }), \quad e^{\frac{1}{2} \lambda \sigma}=\frac{1}{2} \lambda^{2} K r
$$

where

$$
r^{2}=e^{\lambda^{2} K(\lambda) z}
$$

This solution was first found in [3]. Recall that $\lambda<2 \alpha$ when $\Lambda<0$; in particular, there is a fixed point for $\lambda=\lambda_{c}$ when $\Lambda<0$, with fixed-point solution

$$
d s_{d}^{2}=d r^{2}+r^{2} d s_{d-1}^{2}(\text { Mink }), \quad e^{\frac{1}{2} \lambda_{c} \sigma}=\frac{\sqrt{|\Lambda|}}{(d-2)} r .
$$


- Type 2. In this case $\Lambda<0$, necessarily. At this fixed point we have

$$
\sigma=\lambda_{c} \sqrt{|\Lambda|} z+\sigma_{0}, \quad 2 \beta \varphi=\lambda \lambda_{c} \sqrt{|\Lambda|} z+2 \beta \varphi_{0}
$$

for constants $\sigma_{0}$ and $\varphi_{0}$. We may assume that $\lambda \neq \lambda_{c}$ because the coincidence of the fixed points at $\lambda=\lambda_{c}$ means that the fixed point solution is the same as the $\lambda=\lambda_{c}$ case of the Type 1 solution discussed above. For $\lambda \neq \lambda_{c}$ we are not free to choose the constants $\left(\sigma_{0}, \varphi_{0}\right)$ arbitrarily because the constraint (2.3) requires

$$
\lambda \sigma_{0}-2 \beta \varphi_{0}=\log \left[\frac{\left|\left(\lambda^{2}-\lambda_{c}^{2}\right) \Lambda\right|}{2(d-2)}\right] .
$$

However, we may choose

$$
\sigma_{0}=\frac{2}{\lambda} \log \left(\lambda \lambda_{c} \sqrt{|\Lambda|} / 2\right),
$$

without loss of generality, in which case the fixed-point solution is

$$
d s_{d}^{2}=d \rho^{2}+\frac{\lambda^{2}}{\left|\lambda^{2}-\lambda_{c}^{2}\right|} \rho^{2} d \Sigma_{k}^{2}, \quad e^{\frac{1}{2} \lambda \sigma}=\frac{1}{2} \lambda \lambda_{c} \sqrt{|\Lambda|} \rho,
$$

where

$$
\rho^{2}=e^{\lambda \lambda_{c} \sqrt{|\Lambda|} z} .
$$

For $\lambda=0$, the fixed point is in the region of the phase plane with $k=-1$ and we thus recover the $a d S_{d-1} \times \mathbb{R}$ product metric found in the previous section. For $\lambda>\lambda_{c}$ the fixed point is in the $k=1$ region of the phase plane and the fixed point solution is

$$
d s_{d}^{2}=d \rho^{2}+\frac{\lambda^{2}}{\left|\lambda^{2}-\lambda_{c}^{2}\right|} \rho^{2} d s_{d-1}^{2}(d S), \quad e^{\frac{1}{2} \lambda \sigma}=\frac{1}{2} \lambda \lambda_{c} \sqrt{|\Lambda|} \rho .
$$

In the limit as $\lambda \rightarrow \infty$ the $d$-metric becomes a flat static Rindler-type metric that is the analytic continuation of the Milne metric through its cosmological horizon; this is possible because the stress tensor for $\sigma$ is proportional to $1 / \lambda^{2}$.

\subsection{Flat walls}

Flat domain walls, for which the worldvolume geometry is Minkowski, are found by considering $k=0$. In this special case, the equations (2.7) reduce to

$$
\dot{u}=\frac{1}{2}(\lambda v-2 \alpha u) v, \quad \dot{v}=\frac{1}{2}(\lambda v-2 \alpha u) u,
$$

and the constraint is

$$
v^{2}-u^{2}+2 \Lambda=0 .
$$

Solving the constraint by setting

$$
v=\sqrt{|\Lambda| / 2}\left(\xi-\operatorname{sign} \Lambda \xi^{-1}\right), \quad u=\sqrt{|\Lambda| / 2}\left(\xi+\operatorname{sign} \Lambda \xi^{-1}\right),
$$


we find that the equations (4.12) are equivalent to

$$
\dot{\xi}=\frac{1}{4} \sqrt{2|\Lambda|}\left[(\lambda-2 \alpha) \xi^{2}-(\operatorname{sign} \Lambda)(\lambda+2 \alpha)\right] .
$$

For future convenience, we choose to present the solutions for $\lambda=0$ and $\lambda>0$ separately.

\subsection{1 $\lambda=0$}

In this case (4.15) reduces to

$$
\dot{\xi}=-\alpha \sqrt{\mid \Lambda / 2}\left(\operatorname{sign} \Lambda+\xi^{2}\right) .
$$

This is easily solved and leads to the following solutions for $(\varphi, \sigma)$ :

- $\Lambda>0$. In this case

$$
e^{\alpha \varphi}=\frac{1}{2}|\sin (\alpha \sqrt{2 \Lambda} z)|, \quad e^{\alpha \sigma}=|\cot (\alpha \sqrt{\Lambda / 2} z)| .
$$

Formally, this is a periodic solution with period $\pi /[\alpha \sqrt{2 \Lambda}]$ but because $\varphi$ is singular at $z=0$ we should consider $z>0$ and $z<0$ as yielding different solutions. Moreover we may restrict to $\alpha \sqrt{2 \Lambda}|z|<\pi$ as $\sigma$ is singular when $\alpha \sqrt{2 \Lambda}|z|=\pi$. The two solutions with $z>0$ and $z<0$ yield the solutions corresponding to the two branches of the $k=0$ hyperbola.

- $\Lambda<0$. In this case there is a fixed point solution, which we have already discussed. Otherwise, we have

$$
e^{\alpha \varphi}=\frac{1}{2}|\sinh (\alpha \sqrt{2|\Lambda|} z)|, \quad e^{ \pm \alpha \sigma}=|\operatorname{coth}(\alpha \sqrt{|\Lambda| / 2} z)|
$$

For either choice of the sign we have two solutions, corresponding to $z>0$ and $z<0$, since $\varphi$ is singular at $z=0$. These are the two branches of the $k=0$ hyperbola, with $\dot{\varphi}>0$ for $z>0$ and $\dot{\varphi}<0$ for $z<0$. On each branch there are two solutions, apart from the fixed point solution, depending on whether $\dot{\sigma}$ is positive or negative; this corresponds to the choice of sign in (4.18).

\subsection{2 $\lambda>0$}

It is convenient to introduce the quantities

$$
A=\frac{\sqrt{\left|\left(4 \alpha^{2}-\lambda^{2}\right) \Lambda\right|}}{2 \sqrt{2}}, \quad \nu_{ \pm}=\frac{2}{2 \alpha \pm \lambda} .
$$

The solutions can be given jointly for either sign of $\lambda$, according to whether $(\lambda-2 \alpha) \Lambda$ is positive, negative or zero: 
- $\lambda=2 \alpha$. In this case, $\xi=-\alpha(\operatorname{sign} \Lambda) \sqrt{2|\Lambda|} z$, and

$$
e^{2 \alpha \varphi}=z e^{-\Lambda \alpha^{2} z^{2}}, \quad e^{2 \alpha \sigma}=z^{-1} e^{-\Lambda \alpha^{2} z^{2}}
$$

- $(\lambda-2 \alpha) \Lambda<0$. In this case,

$$
\xi=-(\operatorname{sign} \Lambda) \sqrt{\frac{\lambda+2 \alpha}{|\lambda-2 \alpha|}} \tan A z
$$

and the solution is

$$
e^{\varphi}=|\cos A z|^{\nu_{-}}|\sin A z|^{\nu_{+}}, \quad e^{\sigma}=|\cos A z|^{\nu_{-}}|\sin A z|^{-\nu_{+}} .
$$

- $(\lambda-2 \alpha) \Lambda>0$ In this case, there are two solutions of (4.15), in addition to the fixed point solutions already considered:

$$
\begin{aligned}
(i): \xi & =-(\operatorname{sign} \Lambda) \sqrt{\frac{2 \alpha+\lambda}{2 \alpha-\lambda}} \tanh A z, \\
(i i): \xi & =-(\operatorname{sign} \Lambda) \sqrt{\frac{2 \alpha+\lambda}{2 \alpha-\lambda}} \operatorname{coth} A z .
\end{aligned}
$$

These yield the solutions

$$
\text { (i) } \quad \begin{aligned}
e^{\varphi} & =(\cosh A z)^{\nu_{-}}|\sinh A z|^{\nu_{+}} \\
e^{\sigma} & =(\cosh A z)^{\nu_{-}}|\sinh A z|^{-\nu_{+}}
\end{aligned}
$$

and

$$
\text { (ii) } \begin{aligned}
e^{\varphi} & =(\cosh A z)^{\nu_{+}}|\sinh A z|^{\nu_{-}}, \\
e^{\sigma} & =(\cosh A z)^{-\nu_{+}}|\sinh A z|^{\nu_{-}} .
\end{aligned}
$$

\subsection{Generic domain walls}

A generic trajectory in the $(u, v)$ phase-plane is a solution to the differential equation

$$
\left(\beta v^{2}+\frac{1}{2 \alpha^{2}} u^{2}+2 \beta \Lambda-\frac{1}{2} \lambda u v\right) d u+\left(\frac{1}{2} \lambda u^{2}-\alpha u v-\lambda \Lambda\right) d v=0 .
$$

The left hand side is not an exact differential but an integrating factor exists. For $\lambda=0$ we were able to find the integrating factor and hence we were able to find all the trajectories exactly. We have not found the integrating factor for $\lambda>0$, so in this case we must fall back on a qualitative analysis of the phase-plane trajectories. However, given that one has trajectories for any $\lambda$, one can ask what they look like in the limit as $\lambda \rightarrow \infty$. In this limit, an integrating factor is easily found and this yields the curves

$$
C v^{2}-u^{2}+2 \Lambda=0
$$




$$
\Lambda>0,0<\lambda<2 \alpha
$$

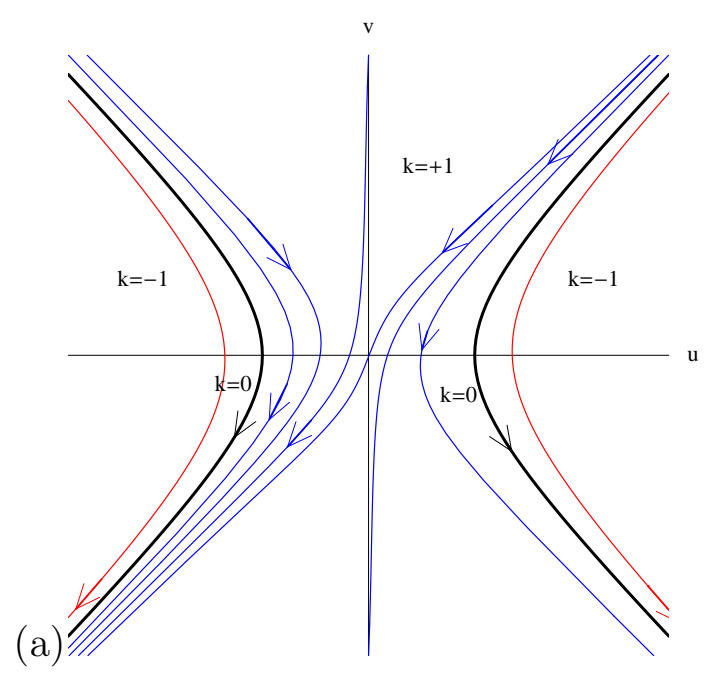

$\Lambda>0, \lambda>2 \alpha$

(b)

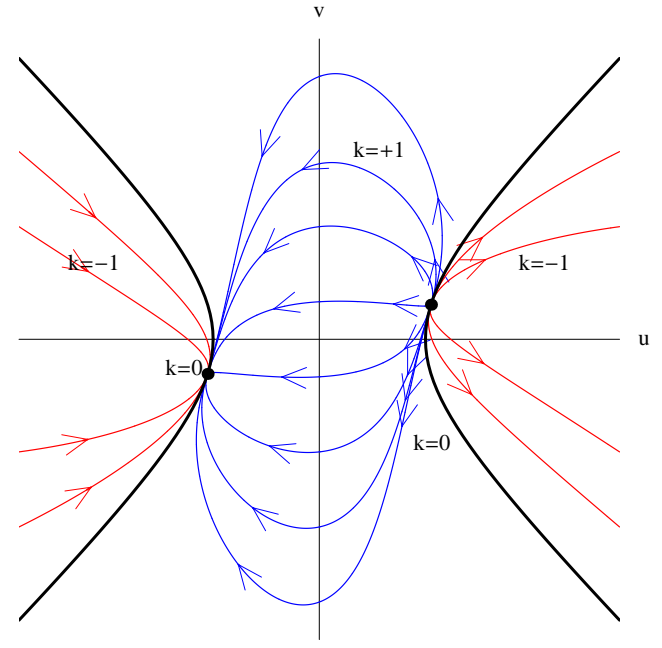

Figure 5: (a) The phase plane topology is the same as for $\lambda=0$ but the lateral symmetry is lost and there are now two trajectories that are asymptotic to the $v$-axis. (b) For $\lambda>2 \alpha$ there are two $k=0$ fixed points, both of which are nodes. All $k=1$ trajectories start at one node and end at the other one.

for some constant $C$, which must be non-negative for $\Lambda<0$ but may be positive or negative for $\Lambda>0$.

The phase-plane plots are essentially the same as those found in the cosmological case [1] for $d=4$ (and $\Lambda>0$, which corresponds here to $\Lambda<0$ ) but with the different interpretation discussed earlier. As there are are never more than four hyperbolic fixed points, the topological structure of the phase-plane is determined unambiguously by these fixed points as long as there are no limit cycles. For $\Lambda>0$ or for $\Lambda<0$ when $\lambda<\lambda_{c}$, any closed curved in the phase plane with unit Poincaré index must cross the $k=0$ hyperbola and so cannot be a limit cycle. Thus a limit cycle is possible only if $\Lambda<0$ and $\lambda>\lambda_{c}$, and any such cycle would have to enclose a $k=1$ fixed point (which is either a node or a focus, as explained below, and hence has unit Poincaré index). We have not seen how to prove that a limit cycle never appears for any $d$ as $\lambda$ is increased indefinitely, but numerical plots for various cases are consistent with the absence of limit cycles. We present a representative selection of $\lambda>0$ phase-plane plots in Figs. 3,4,5. These were obtained numerically for $d=7$ and particular choices of $\lambda$ in the specified ranges. Note the symmetry under reflection through the origin, in all cases. For $\Lambda<0$ and $\lambda>2 \alpha$ the $k=1$ fixed point is either a node or a focus (spiral) depending on $d$ and the precise value of $\lambda$. The details are the same as in the cosmology case [5]. In the notation of this paper one finds that the fixed point is a node for all $d \geq 10$ and for $d<10$ if $\lambda_{c}<\lambda \leq \bar{\lambda}$, where ${ }^{5}$

$$
\bar{\lambda}=\frac{4}{\sqrt{(d-2)(10-d)}} .
$$

\footnotetext{
${ }^{5}$ Note that $\bar{\lambda} \leq 2 \alpha$ with equality for $d=9$.
} 
Otherwise, the $k=1$ fixed point is a focus. However, there is no topological distinction between a focus and a node.

Note that for $\Lambda<0$ and $\lambda<\lambda_{c}$, the phase-plane plot of Fig. 3a shows that there is a one-parameter family of Janus-type solutions that interpolate between the isometric domain wall spacetimes corresponding to the two $k=0$ fixed points. These are deformations of the family of Janus solutions of the $\lambda=0$ case.

\section{Supersymmetry}

For various values of the coupling constant $\lambda$, and choices of the sign of $\Lambda$, the Lagrangian density (1.1) is the consistent truncation of a supergravity Lagrangian density for which the metric and dilaton are the only bosonic fields. In this context one can ask whether any given solution preserves some fraction of the supersymmetry of the supergravity vacuum. A necessary condition for (partial) supersymmetry preservation is the vanishing of the dilatino supersymmetry transformation. This imposes the condition

$$
\left(\Gamma^{\mu} \partial_{\mu} \sigma+2 W^{\prime}\right) \epsilon=0
$$

where $\epsilon$ is the supersymmetry spinor parameter, and $W(\sigma)$ is the superpotential, which must satisfy

$$
\left(W^{\prime}\right)^{2}-\alpha^{2} W^{2}=\frac{1}{2} \Lambda e^{-\lambda \sigma}
$$

The matrices $\Gamma_{\mu}$ obey the Dirac commutation relations in the given background, which is all that we need to know about them, although they may not actually be the Dirac matrices ${ }^{6}$.

Given a metric of the form (2.4), we may choose frame 1-forms

$$
e_{z}=e^{\frac{1}{2} \lambda \sigma} d z, \quad e_{m}=e^{\beta \varphi} \hat{e}_{m}
$$

where $\hat{e}_{m}(m=0,1, \ldots, d-2)$ are a set of frame 1 -forms for the $(d-1)$ metric $d \Sigma_{k}^{2}$ on the wall. In such a frame we have

$$
\Gamma_{\mu}=\left(e^{\frac{1}{2} \lambda \sigma} \Gamma_{z}, e^{\beta \varphi} \hat{\Gamma}_{m}\right)
$$

where $\Gamma_{z}$ is a constant matrix that squares to the identity and anticommutes with the matrices $\hat{\Gamma}_{m}$. Given that $\sigma$ is a function only of $z$, the condition (5.1) now reduces to

$$
\dot{\sigma}= \pm 2 e^{\frac{1}{2} \lambda \sigma} W^{\prime}
$$

If this is satisfied for $\dot{\sigma}=0$ (and hence constant $\sigma$ such that $W^{\prime}(\sigma)=0$ ) then there is no condition on $\epsilon$. Otherwise

$$
\left(1 \pm \Gamma_{z}\right) \epsilon=0
$$

\footnotetext{
${ }^{6}$ They are the Dirac matrices for $d=3$ minimal supergravity, but they are not necessarily irreducible for other odd dimensions (or non-minimal supergravities); for example, $\Gamma_{\mu}=i \sigma_{2} \otimes \gamma_{\mu}$ for $d=5$. In even dimensions $\Gamma_{\mu}$ is the product of $\gamma_{\mu}$ with the chirality matrix, in a Majorana basis.
} 
which implies, in the absence of any further condition on $\epsilon$, that $1 / 2$ supersymmetry is preserved.

We must also take into account the Killing spinor condition

$$
\left(D_{\mu}-\frac{1}{2(d-2)} W \Gamma_{\mu}\right) \epsilon=0,
$$

which arises, in a supergravity context, from the requirement of vanishing gravitino variation. This is equivalent to the equations

$$
\begin{aligned}
{\left[\partial_{z}-\frac{1}{2(d-2)} e^{\frac{1}{2} \lambda \sigma} W \Gamma_{z}\right] \epsilon } & =0 \\
{\left[\hat{D}_{m}+\frac{\beta}{2} e^{\beta \varphi} \hat{\Gamma}_{m}\left(\dot{\varphi} e^{-\frac{1}{2} \lambda \sigma} \Gamma_{z}-2 \alpha W\right)\right] \epsilon } & =0
\end{aligned}
$$

where $\hat{D}_{m}$ is the covariant derivative on spinors restricted to the domain wall, and with respect to the frame 1 -forms $\hat{e}_{m}$. The second of these equations has the integrabilty condition

$$
\dot{\varphi}^{2}=e^{\lambda \sigma}\left[4 \alpha^{2} W^{2}+\frac{k}{\beta^{2}} e^{-2 \beta \varphi}\right] .
$$

There is a further joint integrability condition of equations (5.8). Using (5.9) and

$$
\ddot{\varphi}=\frac{1}{2}(\lambda \dot{\varphi}-2 \alpha \dot{\sigma}) \dot{\sigma}-\frac{k}{\beta} e^{\lambda \sigma-2 \beta \varphi},
$$

which follows from (2.7) and (2.3), this remaining integrability condition can be reduced to

$$
\dot{\sigma}\left(\dot{\sigma}+2 e^{\frac{1}{2} \lambda \sigma} W^{\prime} \Gamma_{z}\right) \epsilon=0 .
$$

This is an identity if $\dot{\sigma}=0$; otherwise it reduces to (5.5) with $\epsilon$ constrained by (5.6). Moreover, (5.9) can be derived by combining (5.2) with the constraint (2.3) and eliminating $W^{\prime}$ from the resulting expression by means of (5.5). Thus, for domainwall solutions of the field equations, the 'dilatino' supersymmetry preserving condition (5.5) is the Killing spinor integrability condition.

For $k=0$ we may choose cartesian coordinates for which $\hat{D}_{m}=\partial_{m}$. In this special case, a spinor satisfying (5.6) will be a function only of $z$, and subject to no further algebraic constraints, iff

$$
\dot{\varphi}=\mp 2 \alpha e^{\frac{1}{2} \lambda \sigma} W \quad(k=0) .
$$

This is of course consistent with the integrability condition (15.9), but also fixes the sign of $\dot{\varphi}$. The Killing spinor itself is given by integration of

$$
\partial_{z} \epsilon=\mp \frac{1}{2(d-2)} e^{\frac{1}{2} \lambda \sigma} W \epsilon .
$$

Application of these results to the problem in hand requires that we find a superpotential $W$ satisfying (5.2). It is instructive to consider first the $\lambda=0$ case. 


\section{$5.1 \lambda=0$}

In this case, (5.2) reduces to

$$
2\left[\left(W^{\prime}\right)^{2}-\alpha^{2} W^{2}\right]=\Lambda .
$$

There are three possible superpotentials, which we consider in turn:

- $2 \alpha W=\sqrt{2|\Lambda|}$.

This applies for $\Lambda<0$. As $W^{\prime}=0$ it is clear from (5.5) that only domain wall solutions with $\sigma=\sigma_{0}$, for constant $\sigma_{0}$, can be supersymmetric for this superpotential, and that in this case the condition for supersymmetry reduces to the Killing spinor conditions (5.8). Writing $\epsilon=\epsilon^{+}+\epsilon^{-}$, where $\Gamma_{z} \epsilon^{ \pm}= \pm \epsilon^{ \pm}$, we find that these conditions become

$$
\partial_{z} \epsilon^{ \pm}= \pm \beta \sqrt{\frac{|\Lambda|}{2}} \epsilon^{ \pm}, \quad \hat{D}_{m} \epsilon^{ \pm}=\frac{\beta}{2} e^{\beta \varphi}(\sqrt{2|\Lambda|} \pm \dot{\varphi}) \hat{\Gamma}_{m} \epsilon^{\mp} .
$$

The first of these equations is solved by

$$
\epsilon^{ \pm}=e^{ \pm \beta \sqrt{|\Lambda| / 2} z} \zeta^{ \pm},
$$

for $z$-independent spinor $\zeta^{ \pm}$satisfying $\Gamma_{z} \zeta^{ \pm}= \pm \zeta^{ \pm}$. The remaining equations then reduce to

$$
\hat{D}_{m} \zeta^{ \pm}=\frac{1}{2} C_{ \pm} \hat{\Gamma}_{m} \zeta^{\mp}
$$

where

$$
C_{ \pm}=\beta(\sqrt{2|\Lambda|} \pm \dot{\varphi}) e^{\beta \varphi \mp \sqrt{2|\Lambda|} z} .
$$

The integrability conditon for (5.17) is

$$
C_{+} C_{-}=-k .
$$

A rescaling of $\zeta^{+}$and $\zeta^{-}$rescales $C_{+}$and $C_{-}$, leaving the product $C_{+} C_{-}$unchanged, so we may choose $C_{+}=-k C_{-}$without loss of generality. This choice leads to

$$
\dot{\varphi}=\mp \sqrt{2|\Lambda|}\left(\frac{e^{\mp \beta \sqrt{2|\Lambda|} z}+k e^{ \pm \beta \sqrt{2|\Lambda|} z}}{e^{\mp \beta \sqrt{2|\Lambda|} z}-k e^{ \pm \beta \sqrt{2|\Lambda|}}}\right) .
$$

This reproduces the solutions of section 3.1 for the three foliations of $a d S_{d}$. Thus, all supersymmetry is preserved by the $a d S_{d}$ solution irrespective of how it is foliated.

- $2 \alpha W=\sqrt{2|\Lambda|} \cosh \alpha \sigma$.

This again applies for $\Lambda<0$ and was considered in [7. From (5.5) we see that only domain wall solutions with

$$
\dot{\sigma}= \pm \sqrt{2|\Lambda|} \sinh \alpha \sigma
$$


can preserve some fraction of supersymmetry. One possibility is $\sigma=0$, in which case we again have $a d S_{d}$ for any $k$ and all supersymmetries are preserved. This case is analogous to the $a d S_{d}$ solution allowed for constant $W$ but with the difference that supersymmetry now requires $\sigma_{0}=0$. Thus, the supersymmetric $a d S_{d}$ solution allowed for this superpotential is less general than that allowed by a constant superpotential. However, we now have the possibility of a supersymmetric solution with non-constant $\sigma$; specifically, supersymmetry requires

$$
e^{\alpha \sigma}= \begin{cases}\mp \operatorname{coth}(\alpha \sqrt{|\Lambda| / 2} z) & \sigma>0 \\ \mp \tanh (\alpha \sqrt{|\Lambda| / 2} z) & \sigma<0 .\end{cases}
$$

This implies

$$
\alpha \sigma= \pm \log |\operatorname{coth}(\alpha \sqrt{|\Lambda| / 2} z)|,
$$

which is precisely the function $\sigma(z)$ for the $k=0$ domain wall solution (4.17), as shown originally in [10].

- $2 \alpha W=\sqrt{2|\Lambda|} \sinh \alpha \sigma$.

This applies for $\Lambda>0$. It is again clear from (5.1) that only domain wall solutions with

$$
\dot{\sigma}= \pm \sqrt{2 \Lambda} \cosh \alpha \sigma
$$

can preserve some fraction of supersymmetry. This implies that

$$
e^{\alpha \sigma}=|\cot (\alpha \sqrt{\Lambda / 2} z)|
$$

which is precisely the function $\sigma(z)$ for the $k=0$ domain wall solution (4.18).

We have now shown, for $\lambda=0$, that for each flat domain wall solution with nonconstant $\sigma$ there is superpotential for which the supersymmetry preserving condition (5.5) is satisfied. This implies that the Killing spinor integrability condition is satisfied too, and Killing spinors satisfying (5.6) are found by integration of (5.13), for $\lambda=0$. For the $k=0$ fixed point solution, which is just $a d S_{d}$ in horospherical coordinates, there are also Killing eigenspinors of $\Gamma_{z}$ with the opposite eigenvalue [22, and all supersymmetries are preserved in this special case. Thus, all $k=0$ solutions preserve at least $1 / 2$ supersymmetry for some choice of $W$.

\section{$5.2 \lambda>0$}

When $(\lambda-2 \alpha) \Lambda>0$, one possible choice of superpotential is

$$
W=K(\lambda) e^{-\frac{1}{2} \lambda \sigma},
$$

where $K(\lambda)$ is the function given in (2.11). For this superpotential the supersymmetry preserving condition (5.5) becomes $\dot{\sigma}=\mp \lambda K(\lambda)$, which is satisfied only at the $k=0$ 
fixed point, as one would expect from the fact that an exponential superpotential is the natural generalization to $\lambda>0$ of the constant superpotential considered above for $\lambda=0$. One may verify that, for the above superpotential, (5.12) is also satisfied by the $k=0$ fixed point solutions of subsection 4.1. so $1 / 2$ supersymmetry is preserved. This is a well-known result in the context of various specific supergravity theories with an exponential superpotential. An example with $\lambda>\lambda_{c}$ is the maximal gauged $\mathrm{d}=8$ supergravity [19] for which the $k=0$ fixed point solution was shown in [20] to preserve $1 / 2$ supersymmetry. There are several cases with $\lambda=\lambda_{c}$, for $d=5,6,7$. An example is the minimal $d=7$ gauged supergravity [21] for which the $k=0$ fixed point solution was shown in [3] to preserve $1 / 2$ supersymmetry. Cases with $\lambda<\lambda_{c}$ arise from toroidal compactification of a higher dimensional model with an adS vacuum 22. An example for which $\lambda$ is arbitrary is $d=3$ adS $N=1$ supergravity coupled to a scalar multiplet, the Lagrangian and supersymmetry transformation rules of which can be found in [23].

Given our results for $\lambda=0$, it would be natural to suppose that there exist $\lambda$ deformations of the $\cosh \alpha \sigma$ and $\sinh \alpha \sigma$ superpotentials for which the non-fixed-point $k=0$ solutions would also be supersymmetric. To investigate this, we differentiate both sides of (5.2) with respect to $\sigma$ and then use (5.2) to eliminate $\Lambda$. We thus find that the function

$$
X(\sigma)=W^{\prime}(\sigma) / W(\sigma)
$$

obeys the first-order ODE

$$
2 X X^{\prime}=(2 X+\lambda)\left(\alpha^{2}-X^{2}\right) .
$$

This equation is obviously solved by $X= \pm \alpha$, but this we discard because it requires $\Lambda=0$. It is also obviously solved by $X=-\lambda / 2$; this yields the superpotential (5.26). Unless $\lambda=2 \alpha$, all other solutions are given by $^{7}$

$$
|X+\lambda / 2|^{2 \lambda}|X-\alpha|^{2 \alpha-\lambda}|X+\alpha|^{-2 \alpha-\lambda}=e^{\left(\lambda^{2}-4 \alpha^{2}\right) \sigma} .
$$

For any of the functions $X(\sigma)$ defined implicitly by this algebraic relation we have the superpotential

$$
W(\sigma)=\exp \left(\int^{\sigma} X(s) d s\right) .
$$

For $\lambda=0$, (5.29) simplifies to

$$
\left|\frac{X-\alpha}{X+\alpha}\right|=e^{-2 \alpha \sigma}
$$

and hence

$$
X=\left\{\begin{array}{ll}
\alpha \tanh (\alpha \sigma) & |X|<\alpha \\
\alpha \operatorname{coth}(\alpha \sigma) & |X|>\alpha
\end{array} \quad(\lambda=0)\right.
$$

\footnotetext{
${ }^{7}$ This is for a specific choice of the integration constant, which we may choose without loss of generality; a change in this constant is equivalent to a shift of $\sigma$, which is equivalent to a scaling of $\Lambda$, but $\Lambda$ cancels from the ratio $W^{\prime} / W=X$. The solution for $\lambda=2 \pi$ can be found too, but it is less illuminating and we omit it.
} 
These yield, respectively, the $\cosh (\alpha \sigma)$ and $\sinh (\alpha \sigma)$ superpotentials discussed in the previous subsection. Note that in both cases $|X| \sim \alpha$ as $|\sigma| \rightarrow \infty$, and that $X$ has either a zero or a pole at $\sigma=0$.

The implications of (5.29) when $\lambda \neq 0$ depend on the sign of $(2 \alpha-\lambda)$ :

- $\lambda<2 \alpha$. In this case $\sigma \rightarrow \infty$ implies either $X \rightarrow \alpha$ or $X \rightarrow-\lambda / 2$, and $\sigma \rightarrow-\infty$ implies $X \rightarrow-\alpha$. There is one solution with $|X|>\alpha$ that yields a superpotential with the same asymptotic behaviour as the $\lambda=0$ superpotential $W \propto \sinh (\alpha \sigma)$, and this superpotential is therefore applicable for $\Lambda>0$.

There are also two solutions with $|X|<\alpha$, one with $X<-\lambda / 2$ and the other with $X<-\lambda / 2$. These yield two superpotentials with the same behaviour for large $|\sigma|$ as the $W \propto \cosh (\alpha \sigma)$ superpotential that is applicable for $\Lambda<0$.

- $\lambda>2 \alpha$. In this case $\sigma \rightarrow \infty$ implies $|X| \rightarrow \alpha$ and $\sigma \rightarrow-\infty$ implies $X \rightarrow-\lambda / 2$. There is one solution with $|X|<\alpha$ and two solutions with $|X|>\alpha$, one with $X>-\lambda / 2$ and the other with $X<-\lambda / 2$.

Observe that the number of possible superpotentials is the same as the number of possible $k=0$ domain-wall solutions, if we ignore the freedom in the choice of integration constants. This is no accident, as we now demonstrate.

Given any of the $k=0$ domain wall solutions of subsection 4.2, we have functions $\varphi(z)$ and $\sigma(z)$. As long as $\dot{\sigma} \neq 0$, we may define a function of $\sigma$ by

$$
W(\sigma)=F(z(\sigma))
$$

where

$$
F(z)=\mp \frac{1}{2 \alpha} e^{-\frac{1}{2} \lambda \sigma(z)} \dot{\varphi}(z)
$$

and $z(\sigma)$ is the inverse function to $\sigma(z)$. By construction, the Killing spinor condition (5.12) is satisfied. We now show that the supersymmetry-preserving condition (5.5) is also satisfied. Using (5.10) for $k=0$, we see that

$$
\dot{F}= \pm \frac{1}{2} e^{-\frac{1}{2} \lambda \sigma} \dot{\sigma}^{2}
$$

and hence that

$$
W^{\prime} \equiv \dot{F} / \dot{\sigma}= \pm \frac{1}{2} e^{-\frac{1}{2} \lambda \sigma} \dot{\sigma}
$$

But this is just (5.5) . Thus, every flat domain wall for which $\dot{\sigma}$ is not identically zero determines a putative superpotential with respect to which it preserves at least $1 / 2$ supersymmetry. We say 'putative' because we have still to see whether the function $W(\sigma)$ that we have defined satisfies (5.2). In fact, this is automatic, as we now show. The conditions (5.5) and (5.12) imply

$$
W^{\prime} / W \equiv X=-\alpha u / v
$$

and hence

$$
X^{\prime} \equiv u^{-1} \dot{X}=\frac{\dot{v}}{v}-\frac{\dot{u}}{u v}
$$


But if $(\dot{u}, \dot{v})$ are given by (4.12) then this equation is equivalent to (5.28), which is itself equivalent to (5.2) for non-zero $W$. Thus, every flat domain wall determines a superpotential with respect to which it preserves at least $1 / 2$ supersymmetry ${ }^{8}$

\section{$5.3 k \neq 0$}

To complete our analysis, we now consider whether solutions of our model for non-flat domain walls can also preserve supersymmetry. We start from the observation that (5.9) is equivalent to

$$
W=\frac{\eta}{2 \alpha} \sqrt{e^{-\lambda \sigma} \dot{\varphi}^{2}-\frac{k}{\beta^{2}} e^{-2 \beta \varphi}}
$$

for some sign $\eta$. Given any domain wall solution with non-zero $\dot{\sigma}$, we can use this equation to define a putative superpotential $W$, using the implicit function $z(\sigma)$ to express $W(\sigma(z))$ as a function of $\sigma$. As for $k=0$, we can now compute $W^{\prime}$. Using (5.10) to eliminate the $\ddot{\varphi}$ term, we find that

$$
W^{\prime}=-\frac{\eta \dot{\sigma} \dot{\varphi} e^{-\frac{1}{2} \lambda \sigma}}{2 \sqrt{\dot{\varphi}^{2}-\frac{k}{\beta^{2}} e^{\lambda \sigma-2 \beta \varphi}}} .
$$

This is consistent with (5.5) iff

$$
\dot{\sigma}\left[\dot{\varphi} \pm \eta \sqrt{\dot{\varphi}^{2}-\frac{k}{\beta^{2}} e^{\lambda \sigma-2 \beta \varphi}}\right]=0 .
$$

As we have assumed that $\dot{\sigma}$ is non-zero, this implies that $\eta=\mp 1$ and that $k=0$, in which case (5.39) reduces to (5.12).

Thus, no non-flat dilaton domain walls with non-constant $\sigma$ can be "supersymmetric" in the sense of this paper. Supersymmetric $k=-1$ dilaton domain walls have been found in $d=5$ supergravity theories 24, 25, but these involve additional scalar fields. The possibility of supersymmetric $k=-1$ domain walls in models with a single scalar field has been studied in [7, via the introduction of an $s u(2)$-valued superpotential, with the conclusion that the $a d S_{d-1} \times \mathbb{R}$ solution (obtained here as the $k=-1$ fixed point solution for $\Lambda<0$ and $\lambda=0$ ) is "fake supersymmetric". The phase-plane analysis shows that there exists a solution that interpolates between the $a d S_{d}$ and $a d S_{d-1} \times \mathbb{R}$ fixed-point solutions. We found this "separatrix wall" solution exactly for $d=3$. There are actually four separatrix wall solutions, corresponding to the four possible trajectories that connect an $a d S_{d}$ fixed point to an $a d S_{d-1} \times \mathbb{R}$ fixed point. This is illustrated for $d=3$ in Fig. 6a.

The phase-plane analysis also shows that there exists a one-parameter family of solutions that interpolates between two isometric $a d S_{d}$ spacetimes. These are the Janus solutions discussed in [7]. There is a Janus solution with a phase-plane trajectory that approaches arbitrarily close to the union of two separatrix trajectories.

\footnotetext{
${ }^{8}$ This conclusion was previously arrived at in [7, where it was also suggested that the construction should apply for arbitrary dilaton potential $V$.
} 

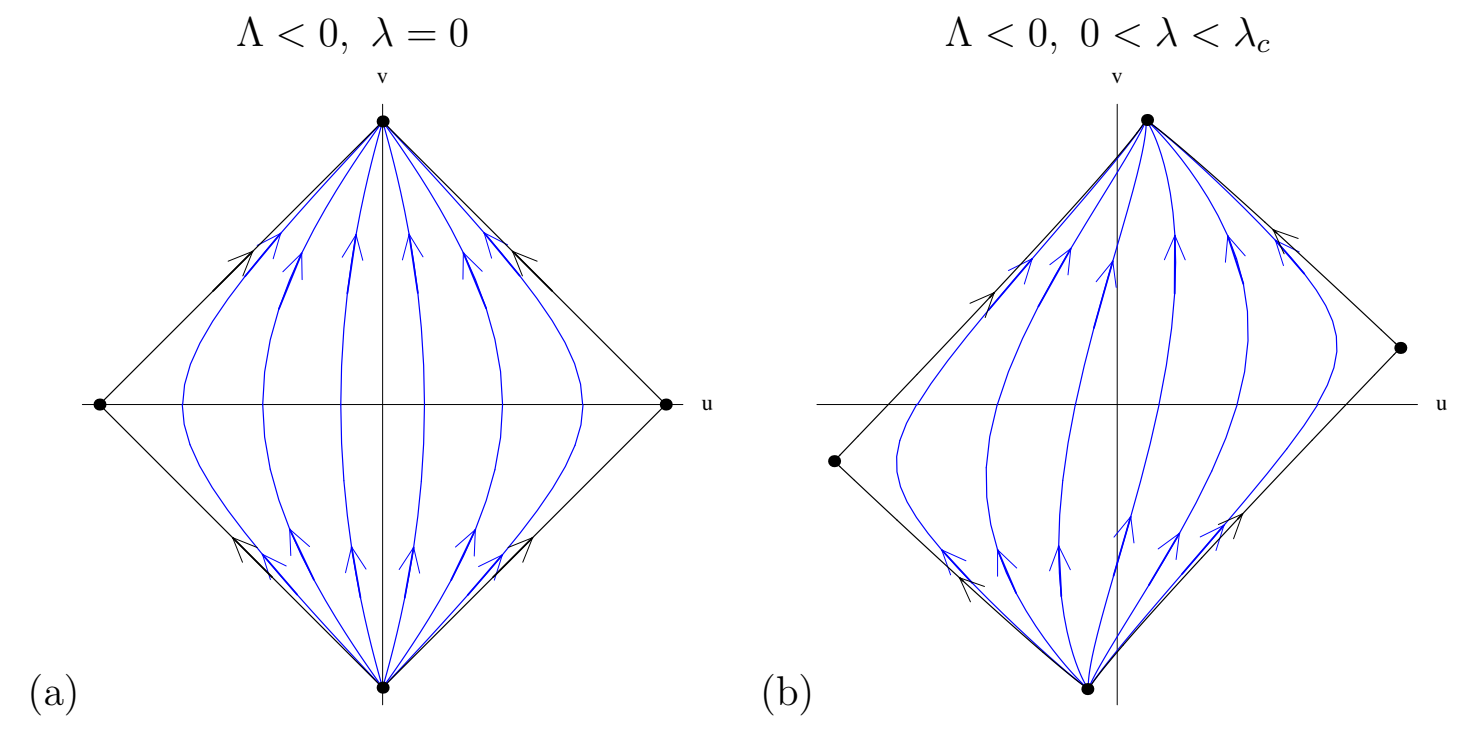

Figure 6: (a) The central region of Fig. 2a for $d=3$. The "Janus" trajectories interpolating between the two $a d S_{3}$ fixed points may come arbitrarily close to one of the $a d S_{2} \times \mathbb{R}$ fixed points. In the limit one gets a trajectory that is the union of two trajectories, each corresponding to the exact "separatrix wall" solution of section [3.3. (b) The small $\lambda$ deformation of the Janus and separatrix trajectories.

This can be viewed as a marginal bound state of two separatrix walls, separated by an arbitrary distance (related to the parameter $c$ in section 3.2). This is a rather unusual state of affairs, reminiscent of the multiple domain wall solutions of certain supersymmetric sigma-models [26]. It suggests a no-force condition that is usually associated with supersymmetry. Indeed, it is claimed in [7] that the Janus solutions are ("fake") supersymmetric, and continuity would then suggest that the separatrix walls have the same property.

As shown in Fig 6b, the same considerations apply for any $\lambda<\lambda_{c}$ in that there still exists a one-parameter family of Janus-type solutions that are asymptotic to both $k=0$ fixed point solutions, but with the difference that these fixed point solutions are no longer adS spaces.

\section{Comments}

We have shown in this paper how the equations governing domain wall solutions of $d$-dimensional gravity coupled to a dilaton with an exponential potential define a family of 2-dimensional autonomous dynamical systems, parametrized by the dilaton coupling $\lambda$, and with a transcritical bifurcation as a function of $\lambda$ when the dilaton potential is negative. This formulation of the problem, which is analogous to the similar formulation of homogeneous and isotropic cosmologies in the same class of models, allows a much more complete understanding of the space of domain-wall 
solutions than has hitherto been possible, particularly for curved domain walls for which the worldvolume geometry is de Sitter $(k=1)$ or anti de Sitter $(k=-1)$.

One difference with the cosmological case is that domain walls can preserve some fraction of supersymmetry, and we have shown that all flat walls are "supersymmetric" with respect to some superpotential $W$ for which $d \log W$ can be found exactly, albeit implicitly, for any $\lambda$. Of course, whether this superpotential actually arises in the context of some supergravity theory is another question, and one that we have not addressed in any detail. We note however that any superpotential is possible in $d=3$ and that there therefore exist supersymmetric domain walls in $d=3$ supergravity models with $\lambda=2 \alpha$, or $\Delta=0$ in the notation of [3, 22]; this is a case for which no domain wall solution, supersymmetric or otherwise, was previously known.

In the case of a pure cosmological constant, corresponding to $\lambda=0$, we found the exact phase-plane trajectories. For $\Lambda<0$ there are two $k=-1$ fixed points, each corresponding to an $a d S_{d-1} \times \mathbb{R}$ solution found in [7]; this is the analog of the Einstein Static Universe that occurs for $\Lambda>0$ in the cosmological case. There are also (isometric) "separatrix wall" solutions with phase-plane trajectories that interpolate between an $a d S_{d-1} \times \mathbb{R}$ fixed points and one of two $a d S_{d} k=0$ fixed-points; we found the exact separatrix trajectories, and the exact solution for $d=3$.

The trajectories that interpolate between the two $a d S_{d}$ fixed points correspond to the one-parameter family of "Janus" solutions of [7], which we have interpreted as marginal bound states of two Separatrix Walls (and the same applies to the "deformed" Janus solutions for $0<\lambda<\lambda_{c}$ ). This interpretation makes physical sense for large separation, but for zero separation the solution degenerates to the $a d S_{d}$ vacuum. This suggests a more precise interpretion of the Janus solutions as separatrix wall/anti-wall bound states. It might seem unlikely that such a configuration could be stable (as is shown in [7]) but the usual intuition need not apply in an adS (or deformed $a d S$ ) background.

Acknowledgements: We are grateful to Eric Bergshoeff, Andres Collinucci and Diederik Roest for the discussions on domain walls that led to the work described here, and for allowing us to take over unchanged some notation and preliminary results from their unpublished notes on this topic. We also thank Mirjam Cvetič and Kostas Skenderis for helpful correspondence. J.S. thanks the following bodies for financial support: the Gates Cambridge Trust, der Studienstiftung des deutschen Volkes, Trinity College Cambridge and PPARC.

\section{References}

[1] J. J. Halliwell, Scalar Fields In Cosmology With An Exponential Potential, Phys. Lett. B 185 (1987) 341.

[2] M. Cvetič, Flat world of dilatonic domain walls, Phys. Rev. Lett. 71, 815 (1993) arXiv:hep-th/9304062. 
[3] H. Lu, C. N. Pope, E. Sezgin and K. S. Stelle, Dilatonic p-brane solitons, Phys. Lett. B 371, 46 (1996) arXiv:hep-th/9511203.

[4] H. Lu, C. N. Pope and K. W. Xu, Liouville and Toda Solutions of M-theory, Mod. Phys. Lett. A 11, 1785 (1996) arXiv:hep-th/9604058.

[5] P. K. Townsend, Cosmic acceleration and M-theory, arXiv:hep-th/0308149.

[6] A. B. Burd and J. D. Barrow, Inflationary Models With Exponential Potentials, Nucl. Phys. B 308, 929 (1988).

[7] D. Z. Freedman, C. Nuñez, M. Schnabl and K. Skenderis, Fake supergravity and domain wall stability, Phys. Rev. D 69, 104027 (2004) arXiv:hep-th/0312055.

[8] W. Boucher, Positive Energy Without Supersymmetry, Nucl. Phys. B 242, 282 (1984).

[9] P. K. Townsend, Positive Energy And The Scalar Potential In Higher Dimensional (Super)Gravity Theories, Phys. Lett. B 148, 55 (1984).

[10] K. Skenderis and P. K. Townsend, Gravitational stability and renormalizationgroup flow, Phys. Lett. B 468, 46 (1999) arXiv:hep-th/9909070.

[11] O. DeWolfe, D. Z. Freedman, S. S. Gubser and A. Karch, Modeling the fifth dimension with scalars and gravity, Phys. Rev. D 62, 046008 (2000) arXiv:hep-th/9909134.

[12] E. A. Bergshoeff, A. Collinucci, D. Roest, J. G. Russo and P. K. Townsend, Classical resolution of singularities in dilaton cosmologies, arXiv:hep-th/0507143.

[13] P. K. Townsend and M. N. R. Wohlfarth, Cosmology as geodesic motion, Class. Quant. Grav. 21 (2004) 5375 arXiv:hep-th/0404241.

[14] P. Glendinning, Stabilty, instability and chaos: an introduction to the theory of nonlinear differential equations, (CUP, 1994).

[15] A. Karch and L. Randall, Locally localized gravity, JHEP 0105, 008 (2001) arXiv:hep-th/0011156.

[16] D. Bak, M. Gutperle and S. Hirano, A dilatonic deformation of AdS(5) and its field theory dual, JHEP 0305, 072 (2003) arXiv:hep-th/0304129.

[17] G. Lopes Cardoso, G. Dall'Agata and D. Lüst, Curved BPS domain wall solutions in five-dimensional gauged supergravity, JHEP 0107, 026 (2001) arXiv:hep-th/0104156.

[18] M. Alishahiha, A. Karch, E. Silverstein and D. Tong, The $d S / d S$ correspondence, AIP Conf. Proc. 743, 393 (2005) arXiv:hep-th/0407125.

[19] A. Salam and E. Sezgin, D = 8 Supergravity, Nucl. Phys. B 258, 284 (1985). 
[20] H. J. Boonstra, K. Skenderis and P. K. Townsend, The domain wall/QFT correspondence, JHEP 9901, 003 (1999) arXiv:hep-th/9807137.

[21] P. K. Townsend and P. van Nieuwenhuizen, Gauged Seven-Dimensional Supergravity, Phys. Lett. B 125, 41 (1983).

[22] H. Lu, C. N. Pope and P. K. Townsend, Domain walls from anti-de Sitter spacetime, Phys. Lett. B 391 (1997) 39 arXiv:hep-th/9607164.

[23] B. de Wit, H. Nicolai and H. Samtleben, Gauged supergravities in three dimensions: A panoramic overview, arXiv:hep-th/0403014.

[24] G. L. Cardoso, G. Dall'Agata and D. Lüst, Curved BPS domain walls and $R G$ flow in five dimensions, JHEP 0203, 044 (2002) arXiv:hep-th/0201270.

[25] K. Behrndt and M. Cvetič, Bent BPS domain walls of $D=5 N=2$ gauged supergravity coupled to hypermultiplets, Phys. Rev. D 65, 126007 (2002) arXiv:hep-th/0201272.

[26] J. P. Gauntlett, D. Tong and P. K. Townsend, Multi-domain walls in massive supersymmetric sigma-models, Phys. Rev. D 64, 025010 (2001) arXiv:hep-th/0012178. 